\title{
Digital inkjet functionalization of water-repellent textile for smart textile application
}

\author{
Junchun $\mathrm{Yu}^{1, *}$ (D), Sina Seipel ${ }^{1}$ (D), and Vincent A. Nierstrasz ${ }^{1}$ (1) \\ ${ }^{1}$ Textile Materials Technology, Department of Textile Technology, Faculty of Textiles, Engineering and Business, University of Borås, \\ 50190 Borås, Sweden
}

Received: 1 May 2018

Accepted: 29 May 2018

Published online:

14 June 2018

(C) The Author(s) 2018

\begin{abstract}
Digital inkjet printing is a production technology with high potential in resource efficient processes, which features both flexibility and productivity. In this research, waterborne, fluorocarbon-free ink containing polysiloxane in the form of micro-emulsion is formulated for the application of water-repellent sportsand work wear. The physicochemical properties of the ink such as surface tension, rheological properties and particle size are characterized, and thereafter inkjet printed as solid square pattern $(10 \times 10 \mathrm{~cm})$ on polyester and polyamide 66 fabrics. The water contact angle (WCA) of the functional surfaces is increased from $<90^{\circ}$ to ca. $140^{\circ}$ after 10 inkjet printing passes. Moreover, the functional surface shows resistance to wash and abrasion. The WCA of functional surfaces is between $130^{\circ}$ and $140^{\circ}$ after 10 wash cycles, and is ca. $140^{\circ}$ after $20000 \mathrm{rev}-$ olutions of rubbing. The differences in construction of the textile as well as inkfilament interaction attribute to the different transportation behaviors of the ink on the textile, reflected in the durability of the functional layer on the textile. The functionalized textile preserves its key textile feature such as softness and breathability. Inkjet printing shows large potential in high-end applications such as customized functionalization of textiles in the domain of smart textiles.
\end{abstract}

\section{Introduction}

In the past decades, inkjet printing was recognized as an emerging production technology because of its manufacturing capabilities. Inkjet printing is applied in various applications such as micro-manufacturing, photovoltaics, electrochemical sensors and ceramic tile [1-4]. Gradual development of the inkjet printing technology [5] has now come to a stage where it is accurate and fast enough to compete as an alternative method for rapid printing, overall coating and periodic micro-patterning.

Inkjet printing targeting various functional applications has been investigated intensively on non-absorbent substrates, such as glass, silicon wafer and polymer film [2]. Selective and mask-free deposition of functional materials is particularly important since the price of functional material is often high and the positioning of the material is critical in the field of microelectronics [6-10]. For example, inkjet printing

Address correspondence to E-mail: Junchun.yu@hb.se 
was applied to manufacture conductive electrodes $[6,7]$, solar cell components [9], and to deposit precursor particles [10]. The selective deposition of functional fluids and the digitalized process made inkjet printing a very versatile and flexible fabrication technology driven by the application demands [10]. Furthermore, nanomaterials and/or functional materials that are compatible with the ink formulation can boost the performance of functional fluids with properties such as super-hydrophobicity/selfcleaning [11, 12], anti-bacterial and electrical conductivity [13]. Nanoparticles of $\mathrm{SiO}_{2}$ [14], $\mathrm{TiO}_{2}, \mathrm{ZnO}$ [15], and Ag [13, 16], functional materials such as polysiloxane [12, 14], polysilane [12] and poly(3,4ethylenedioxythiophene):poly(styrene sulfonate) (PEDOT:PSS) [17] are commonly involved. The efficient material usage, high precision and flexible production with high productivity made inkjet printing a potential method for resource efficient, high-end smart textile manufacturing. Moreover, inkjet printing could be beneficial to maintain the original characteristics of the carrying porous material, e.g., the softness in hand feeling and breathability of textile due to deposition of materials in $\mathrm{pL}$ range [18].

Water repellency, or sometimes extended to superhydrophobicity, is an important function on technical textile to obtain self-cleaning and stain-repellent properties [11, 14, 19], especially for sports- and work wear. This function can be achieved by applying low surface energy compounds and introducing surface roughness via dip-coating, padding, knife coating or spray coating, etc. Liu et al. [1] investigated a singleside super-hydrophobic finishing on cotton by bladecoater with fluoropolymer foam. A water contact angle (WCA) higher than $150^{\circ}$ and hysteresis lower than $15^{\circ}$ were achieved. Zeng et al. [20] reported a one-pot coating solution consisting of SU-8 (a negative photoresist), fluoroalkyl silane and silica nanoparticles to tune WCA of cotton from $<90^{\circ}$ to $160^{\circ}$. The coating is cured by UV light and is durable to withstand 100 laundry cycles. In other researches, surface roughness is combined with hydrophobic substrates or with hydrophobic top-coatings to achieve hydrophobic/super-hydrophobic surfaces without fluorocarbon. Karim et al. [21] inkjet-printed hydroxyl functional polystyrene nanoparticles (emulsion) on polyester in order to introduce surface roughness, which boost the WCA of the surface from $<90^{\circ}$ to $143^{\circ}$. Liu et al. [22] used polydopamine@octadecylamine

(PDA@ODA)

nanocapsules to functionalize polyester fabric. The functionalized surface has WCA of $145^{\circ}$, and the water repellency can be healed by heating. Even though fluorocarbon $\left(\mathrm{C}_{x} \mathrm{~F}_{y}\right)$ in general shows excellent water and oil repellency among known chemical compounds, the bio-accumulative and persistence of fluorocarbon in man and everywhere else in nature make it unacceptable toward the environmental and sustainable development of modern society [23]. Perfluorooctanoic acid (PFOA), its salts and PFOArelated substances, one type of fluorocarbon (socalled C8), is restricted in EU [24]. The industry thereafter moved toward relatively short chain $\mathrm{C} 6$ and C4. However, the performance of $\mathrm{C} 6$ and $\mathrm{C} 4$ is worse than $\mathrm{C} 8$, and the bioaccumulation of persistence organic compound remains [25].

In this study, we investigated an alternative solution that stresses a resource efficient process, inkjet printing and environment-friendly polysiloxane ink, to replace conventional large run machineries with fluorocarbon chemistry. Polysiloxane used in this work is a linear-type polysiloxane (estimated as $M_{\mathrm{w}} \approx 70000$ and $n \approx 900$, defined as middle-high molecular weight polysiloxane by Mojsiewicz-Pienkowska et al. [26]) which does not pose threat to organisms via the respiratory system (when $n>4$ ) and therefore is favorable for textile application. Furthermore, middle-high molecular weight polysiloxane features low toxicity and does not bioaccumulate once it enters the environment according to existing studies [26].

Despite desired functionalities, the fastness properties of functionalized textile toward wash and abrasion are a fundamental challenge [27]. Zhou et al. [28] demonstrated a durable super-hydrophobic $\mathrm{SiO}_{2} / \mathrm{PDMS}$ nanocomposite coating on polyester fabrics by dip-coating. The WCA of the functional layer maintained almost the same $\left(\sim 170^{\circ}\right)$ after 500 wash cycles or after abrasion testing (Martindale method) of 28000 cycles under $12-\mathrm{kPa}$ load. Liu et al. [1] introduced a cross-linking agent, and therefore, the covalently bonded functional layer is capable to stand 20 wash cycles without significant changes in WCA (decreases from $153^{\circ}$ to $148^{\circ}$ ).

In this research, we used inkjet printing to deposit water-repellent ink on polyester and polyamide 66, which is commonly used in the application of functional (technical) textile. There is a lack of publication concerning hydrophobic functionalization by inkjet 
printing on a porous and absorbent (textile) substrate. We focus on the inkjet formulation, the inkjet printing process and ink-substrate interaction for the application of functional textiles in sports- and work wear.

\section{Materials and methods}

\section{Materials}

Raw polysiloxane micro-emulsion dispersion in water (Dow Corning Corporation) was kindly supplied by Univar Inc. The substrates used for inkjet printing were $100 \%$ plain woven polyester fabric (PET, FOV Fabrics AB, Sweden) and $100 \%$ plain woven polyamide 66 fabric (PA, FOV Fabrics AB, Sweden). The PET fabric has a zero twist two ply yarn warp (336 dtex) and weft of 368 dtex and a weight of $145 \mathrm{~g} \mathrm{~m}^{-2}$. The weft/warp count is $22 / 20 \mathrm{~cm}^{-1}$ for PET. The PA fabric has a warp and weft of 188 dtex and a weight of $118 \mathrm{~g} \mathrm{~m}^{-2}$. The weft/warp count is $27 / 39 \mathrm{~cm}^{-1}$ for PA. The fabrics were received as washed and preset from the producer and thereafter soaked and rinsed by deionized water and iron dried before inkjet printing. Reference PET and PA samples with the same construction but finished with fluorocarbon by exhaustion were provided by FOV Fabrics $A B$ and used to compare with the sample functionalized by inkjet printing. The average pore size of PET and PA was 17.7 and $11.2 \mu \mathrm{m}$, respectively, which are measured by a liquid-liquid type porosimeter (PSM 165, Topas $\mathrm{GmbH}$ ).

The polysiloxane dispersion is chosen based on the molecular weight and architecture of polysiloxane, the carrying fluid and the rheological property of the dispersion. A waterborne dispersion containing linear polysiloxane with viscosity from a few to a few tens of $\mathrm{mPa} s$ which is close to the inkjet printing profile of print head is preferred to formulate the ink. Two types of polysiloxane dispersion, one contains amino functional dimethyl polysiloxane $\left(M_{\mathrm{w}}: 70000\right.$, CAS 71750-79-3) and the other contains methylamino siloxane with glycidyl trimethylammonium chloride (CAS 495403-02-6), are evaluated (Supplementary information, SI). It is convenient to formulate inkjet inks using existing polysiloxane dispersion which is available with industry-scale amount. The pre-examination showed that the inkjet-printed amino functional dimethyl polysiloxane dispersion had higher WCA than methylamino siloxane with glycidyl trimethylammonium chloride dispersion (SI, Fig. S1d). Therefore, amino functional dimethyl polysiloxane dispersion (so-called the functional ink later on) is chosen as the focus of hydrophobic component in this study. The amino functional dimethyl polysiloxane dispersion was used as it is after inkjet profile characterization.

\section{Methods}

The rheological properties of the functional ink were measured by a rheometer (Physica MCR500, Paar Physica) with a double gap cylindrical cell. The ink formulation was measured: (a) on heating from 15 to $40{ }^{\circ} \mathrm{C}$ at a constant shear rate of $10000 \mathrm{~s}^{-1}$; and (b) at a shear rate increasing from 0 to $10000 \mathrm{~s}^{-1}$ at $25^{\circ} \mathrm{C}$. The viscosity was acquired at highest measurable shear rate of the instrument at $10000 \mathrm{~s}^{-1}$. The estimated shear rate at the nozzle tip $(\varepsilon)$ of Dimatix print head could reach $\sim 400000 \mathrm{~s}^{-1}$ by using $\varepsilon=v / D$ [29] (drop velocity, $v$, is $8 \mathrm{~m} \mathrm{~s}^{-1}$ and diameter of a nozzle, $D$, is $21 \mu \mathrm{m}$ ).

The surface tension of the ink was measured using an optical tensiometer (Attension Theta, Biolin Scientific). The surface tension of the inks was measured by pendant drop method with an ink drop volume of $4 \mu \mathrm{L}$ and reported in average of three independent measurements. The prepared ink formulation was filtrated through a nylon syringe filter with a pore size of 0.45 and $0.2 \mu \mathrm{m}$, respectively, to qualitatively evaluate the particle size. Still, in order to eliminate agglomeration of particles in the orifice or the nozzle channel, the functional ink was filtered through a nylon syringe filter with a pore size of $0.45 \mu \mathrm{m}$ before loading into the print head.

Inkjet printing was performed using a Xennia Carnelian 100 inkjet development and analysis system equipped with a piezoelectric type, Dimatix Sapphire QS-256/10 AAA print head. The print head features a fundamental printable drop size of $10 \mathrm{pL}$. Solid square pattern $(10 \times 10 \mathrm{~cm})$ and custom-designed patterns were inkjet printed at a resolution of 300 dpi on PET and PA substrates by multi-pass. Thereafter, the inkjet-printed samples were heated in an oven at $150{ }^{\circ} \mathrm{C}$ for $5 \mathrm{~min}$ to dry the functional ink. The dried samples that could still be hydrophilic at the functional surface were immersed in excessive amount of water to remove the water-soluble component in the ink. Eventually the samples were air- 
dried, and the functional surface became hydrophobic.

WCA was measured by sessile drop method with the optical tensiometer (Attension Theta). Three to five random spots on a textile substrate were selected to represent the surface property and measured with a water drop volume of $3 \mu \mathrm{L}$. For a hydrophobic textile surface, the WCA was read $3 \mathrm{~s}$ after the water droplet was stabilized on the substrate. The observed WCA reduces with time for a hydrophilic textile material. Therefore, the WCA was read immediately once the water droplet landed on the substrate. All samples were air-dried in ambient condition with a temperature of $20 \pm 2{ }^{\circ} \mathrm{C}$ and a relative humidity of $20-30 \pm 3 \%$ for $24 \mathrm{~h}$ and ironed prior to measurement. ASTM D5725-99 suggests a repeatability of 7\% of WCA within a laboratory on absorbent substrates; therefore, we only distinguish the result when the WCA difference is larger than $10^{\circ}$ [30].

The wash fastness was tested according to ISO 6330, with type A machine and procedure $4 \mathrm{~N}$. The abrasion test was performed using a Martindale 2000 abrasion tester (Cromocol Scandinavia $\mathrm{AB}$ ) at standard climate with a temperature of $20 \pm 2{ }^{\circ} \mathrm{C}$ and a relative humidity of $65 \pm 2 \%$. The tests were performed according to ISO 12947-2:1998/Cor.1:2002 but modified to investigate the hydrophobicity of the functional layer. The PET and PA samples were conditioned in standard climate for at least $18 \mathrm{~h}$ and subsequently rubbed at 3000, 5000, 10000 and 20000 revolutions, respectively, with a load of $12 \mathrm{kPa}$. The WCA of functional surface was measured at five random locations on each sample after wash or abrasion tests. Scanning electron microscope (SEM) was performed on JEOL JSM-6301F, and EDX analysis was conducted on a Zeiss Supra 60 VP SEM with EDX probe.

\section{Results and discussion}

Polysiloxane was chosen as fluorocarbon-free and water-repellent compound in the functional ink. As shown in Fig. 1a, the viscosity of the functional ink was compared with one type of Dimatix test ink. The viscosity of the functional ink shows a rather flat temperature dependence compared to Dimatix test ink. The viscosity of the functional ink decreases slightly from 11.7 to $9.5 \mathrm{mPa} \mathrm{s}$ from 15 to $40{ }^{\circ} \mathrm{C}$ (comparing to 19.6 to $11.9 \mathrm{mPa} s$ for Dimatix test ink), and has a viscosity of $9.9 \mathrm{mPa} \mathrm{s}(13.6 \mathrm{mPa}$ s for Dimatix test ink) at chosen inkjet temperature of $35^{\circ} \mathrm{C}$. Furthermore, as shown in Fig. 1b, both inks show a Newtonian behavior at a shear rate scan $\left(0-10000 \mathrm{~s}^{-1}\right)$. The functional ink has a viscosity of $9.8 \mathrm{mPa} s$ at $100 \mathrm{~s}^{-1}$ (14.8 mPa s for Dimatix test ink) and $10.1 \mathrm{mPa} s$ at $10000 \mathrm{~s}^{-1}(14.6 \mathrm{mPa}$ s for Dimatix test ink) at $25{ }^{\circ} \mathrm{C}$. The Newtonian behavior of the fluid and weak temperature-dependent viscosity of the ink suggested that it is reasonable to present the viscosity of $9.9 \mathrm{mPa} \mathrm{s}$ of functional ink at $10000 \mathrm{~s}^{-1}$ (high shear rate) and $35{ }^{\circ} \mathrm{C}$ (inkjet printing temperature) for ink characterization at inkjet printing condition. The presented viscosity of $9.9 \mathrm{mPa} s$ of functional ink fits the inkjet printable range, i.e., from 8 to $14 \mathrm{mPa}$.

The surface tension and particle size were analyzed by pendant drop method and filtration test, respectively. The functional ink has a surface tension of $23.9 \mathrm{mN} \mathrm{m}^{-1}$, which is slightly below but still around the typically acceptable surface tension for inkjet printing between 25 and $35 \mathrm{mN} \mathrm{m}^{-1}$ [3]. The transparent functional ink containing polysiloxane in the form of a micro-emulsion should have a particle size distribution between 1 and $100 \mathrm{~nm}$. The stated particle size $(15 \mathrm{~nm})$ is considerably below $200 \mathrm{~nm}$, which is an experienced particle size limitation to avoid agglomeration of particles in the printing nozzle channel [31]. The functional ink was able to filter through nylon syringe filters with pore sizes of 0.45 and $0.2 \mu \mathrm{m}$, respectively, which confirmed that the functional particles are compatible with the inkjet printing process. The results obtained from measurements of viscosity, surface tension and particle size suggested that the functional ink fulfilled the print head specifications.

The functional ink was inkjet printed as solid square $(10 \times 10 \mathrm{~cm})$ or as other customized pattern at a resolution of $300 \mathrm{dpi}$ by printing of 1, 3, 5 and 10 passes. The hydrophobicity of inkjet-printed samples was characterized by WCA measurements. As shown in Fig. 2a, untreated PET and PA woven fabrics showed overall hydrophilic surfaces with WCA $<$ $90^{\circ}$, even though PET fiber is inherently hydrophobic but PA fiber is inherently hydrophilic. The inkjet functionalized surfaces showed significantly increased WCA. The one-pass printed PET $($ PET $\times 1)$ and PA $(\mathrm{PA} \times 1)$ already show that WCA increased from $<90^{\circ}$ to more than $130^{\circ}$. With increased amount of functional inks, the 10-pass printed PET (PET $\times$ 


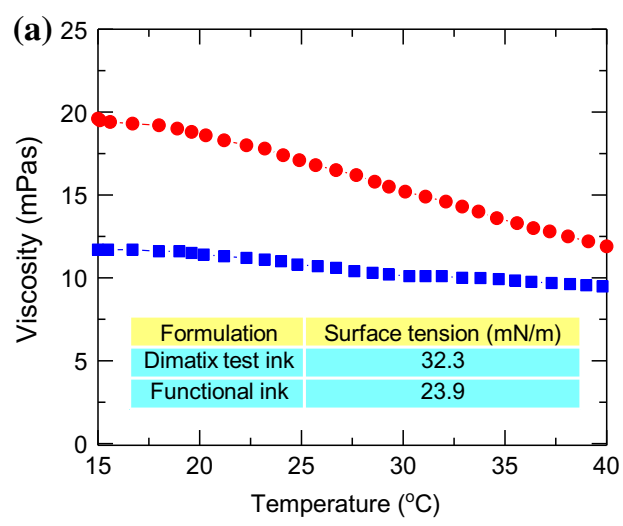

Figure 1 Rheological properties and surface tension of functional and Dimatix test ink. a Viscosity of functional (blue squares) and Dimatix test (red circles) ink measured on heating from 15 to $40{ }^{\circ} \mathrm{C}$ at a shear rate of $10000 \mathrm{~s}^{-1}$, inset: surface tension of

10) and PA $(\mathrm{PA} \times 10)$ show a progressively improved WCA to $136^{\circ}$ and $137^{\circ}$, respectively. In comparison, the reference PET and PA with fluorocarbon finishing by exhaustion have a WCA of $143^{\circ}$ and $140^{\circ}$, respectively. The inkjet-printed functional surface has slightly lower WCA but close to the WCA of a fluorocarbon-finished surface. It is interesting to notice that the hydrophobic functionalized textile shows higher WCA than literature reported on smooth, solid form polysiloxane film $\left(\sim 110^{\circ}\right)$ [32]. This indicates that the surface roughness of the textiles could be equivalent to a periodic pitch between 40 and $50 \mu \mathrm{m}$, in comparison with laser etched polysiloxane with periodic pitch pattern reported by Jin et al. [32]. Moreover, the inkjet-printed samples are all able to hold the water and other water-based droplets such as milk, coffee and blue-colored ink on the surface for more than $10 \mathrm{~min}$ without visually wetting the surface (Fig. 2d). A drop testing kit with various mixtures of isopropanol and water $(0,2,5,10$, 20,30 and $40 \mathrm{vol} \%$ of isopropanol/water mixture) supports the WCA result (SI, Table S2).

SEM was applied to study the morphology of the functional layer. As shown in Fig. 5a, d the functional ink formed a thin layer wrapped around the textile filament. Besides, the functional ink formed uneven circular layers on the surface of PA filament (Fig. 5d), so-called coffee-stain effect after drying of the functional ink [3]. The formation of coffee-stain effect on the PA, which, however, is less obvious on PET and indicates that the inkjet-printed ink was spread more evenly on PET. Energy-dispersive X-ray spectroscopy (EDX) analysis was performed on PA $\times 10$ sample.

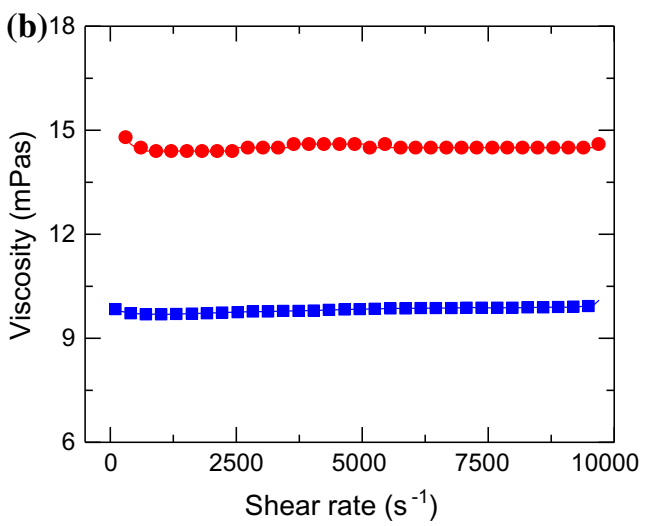

functional and Dimatix test ink; $\mathbf{b}$ viscosity of functional (blue squares) and Dimatix test (red circles) ink measured at increasing shear rate from 100 to $10000 \mathrm{~s}^{-1}$ at $25{ }^{\circ} \mathrm{C}$.

As shown in EDX spectrum in Fig. 5h, $\mathrm{O}$ and $\mathrm{Si}$ were the main element detected which are the components of the functional ink $(\mathrm{O}$ could contribute from $\mathrm{PA}$ substrate as well but less likely due to detectable depth of EDX). Element mapping of Si by EDX was also performed locally on one filament of the PA $\times 10$ (SI, Fig. S5a). Accumulation of Si was detected as layer wrapped around the filament. The above results support that the hydrophobicity of the PET and PA surface is introduced by covering the surface with a thin layer of polysiloxane that is the active compound in the ink formulation.

Inkjet printing can deposit functional fluid on demand. The SEM images in Fig. 3a characterized the inkjet printing boundary of the solid square of PET $\times 10$ sample. The side with smooth and dark appearance is the side with deposited functional ink which is clearly separated from the untreated PET side with rougher and brighter appearance. Moreover, logos of University of Borås and FOV fabrics (original pattern in SI, Fig. S4a, b) were inkjet printed and thereafter sprayed with blue-colored ink all over the surface to test the water repellency. As shown in Fig. 2c, the area without functional ink absorbed the blue-colored ink and therefore revealed the logo. The residual blue color on the functional surface could be rinsed away easily by running water (SI, Fig. S4c, d). Because of the advantage of digitalized communication, inkjet printing combined with functional ink could be applied as customized patterning tool with emphasis on both production capacity and flexibility for the textile sector. Moreover, inkjet printing is a resource efficient process where only materials 

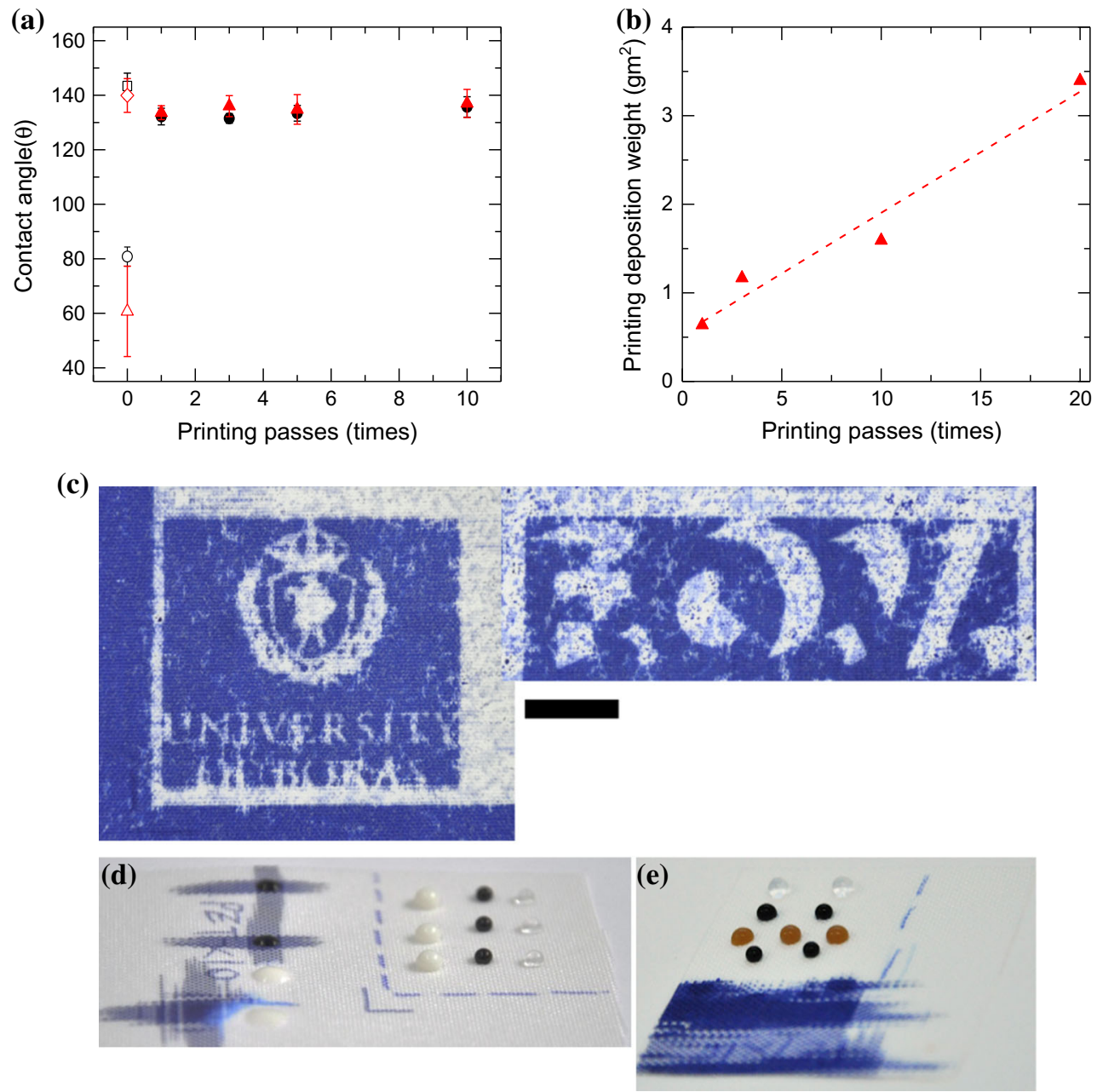

Figure 2 a Water contact angle of PET (circle) and PA (triangle) untreated samples and after inkjet functionalization of hydrophobic ink. The PET (black circles) and PA (red triangles) samples were inkjet printed up to 10 passes $(\times 10)$; untreated PET (open circles) and PA (open red triangles), reference PET (open squares) and PA (open red diamonds) with fluorocarbon finishing were presented. The error bar is the standard deviation from three to five independent measurements. $b$ The weight of the deposited functional material as function of printing passes on PA (red

necessary are deposited. The samples before and after inkjet printing were weighed to calculate the amount of functional material. As shown in Fig. 2b, $3.4 \mathrm{~g} \mathrm{~m}^{-2}$ of functional material were deposited after 20 times or $1.6 \mathrm{~g} \mathrm{~m}^{-2}$ after 10 times inkjet printing. The thickness of the inkjet-printed 10-layer sample is calculated as $1.6 \mu \mathrm{m}$ (density of $1 \mathrm{~g} \mathrm{~cm}^{-3}$ ). Inkjet printing has advantage to localize the deposition of materials on textile. The cross-sectional view of PET $\times 10$ sample (Fig. $3 b$, see arrow) suggested that most of the functional ink was kept at the top surface triangles). The red dashed line is the linear fit of the data. c Demonstration of water repellency of custom-designed pattern on PA $\times 10$ sample to blue-colored ink. The logos of University of Borås and FOV fabrics are inkjet printed (with original pattern in SI, Fig. S4a, b). Demonstration of water repellency of the functional surface to water, milk, coffee and blue-colored droplets. d PET $\times 10$ sample, and e PET $\times 10$ Wash $\times 10$ sample. The blue pen marked area is functionalized with water repellency. The scale bar in the figure is $1 \mathrm{~cm}$.

of the fabric and did not penetrate through the fabric (SEM in SI, Fig. S3a, b). The observation in microstructure was supported by WCA measurement that the printed front side of PET $\times 1$ after 10 washes showed WCA of $\sim 11^{\circ}$ higher than printed backside surfaces. It is good to note that the PET and PA used in this work are not pre-treated to promote surface absorption. Nechyporchuk et al. [13] found that the strike through of inkjet-printed pigment colorants on cotton was ca. $50 \%$ of the thickness of fabric and can be further constrained to the surface 


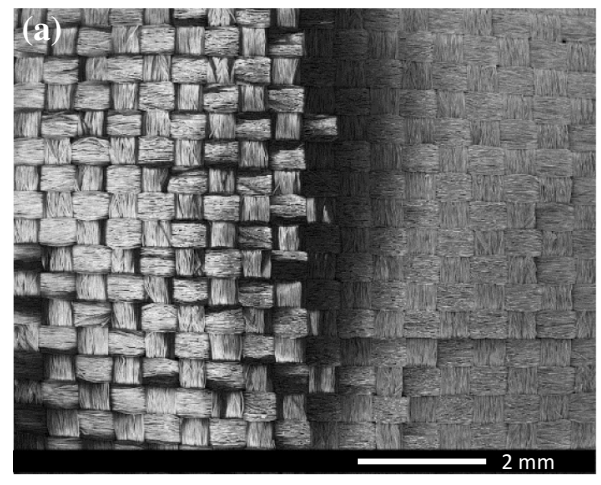

Figure 3 SEM images of inkjet-printed ink on PET substrate $($ PET $\times 10)$. a Top-view of inkjet-printed functional ink boundary. The left side with brighter appearance is untreated PET, whereas the right side with darker appearance is the inkjet functionalized

with a porous top coating. Inkjet printing has the advantage of efficient materials usage in comparison with other coating technologies. It is only possible to deposit the same amount of materials homogeneously all over the bulk textile by dip-coating, or other dipping methods. For single-side deposition technology such as knife coating, Liu et al. [1] demonstrated a fluorocarbon foam, which can typically apply $1.8 \mathrm{~g} \mathrm{~m}^{-2}$ on textile. This amount of material is slightly higher than the case for 10 passes of inkjet printing. However, other components such as rheology modifiers needed to be added in the formulation but was removed after deposition. For formulation with high solid content (> ca. $50 \mathrm{wt} . \%$ ), high viscosity (a few tens of $\mathrm{Pa}$ s) and low shear rate $\left(\sim 2000 \mathrm{~s}^{-1}\right)$ in knife coating, the materials applied (processing speed dependent) are often higher than a few tens of grams per square meter [33, 34]. Although dip-coating and knife coating hypothetically are flexible in production length, inkjet printing still has the advantage to efficiently deposit costly functional materials. Thanks to the precisely controllable deposition of ink volume in $\mathrm{pL}$ range, the key textile features such as tactile feeling and breathability are not affected after functionalization. As shown in the SEM pictures (Fig. 5), the inkjet-printed functional ink formed a thin layer that does not block the inter-yarn pores of the woven textiles, therefore maintaining the tactile feeling and breathability of the textile after inkjet functionalization.

The durability of the functional layer toward wash and abrasion is assessed to simulate the usage of functional textiles in a daily environment. The functionalized textiles were washed at $40{ }^{\circ} \mathrm{C}$ according to

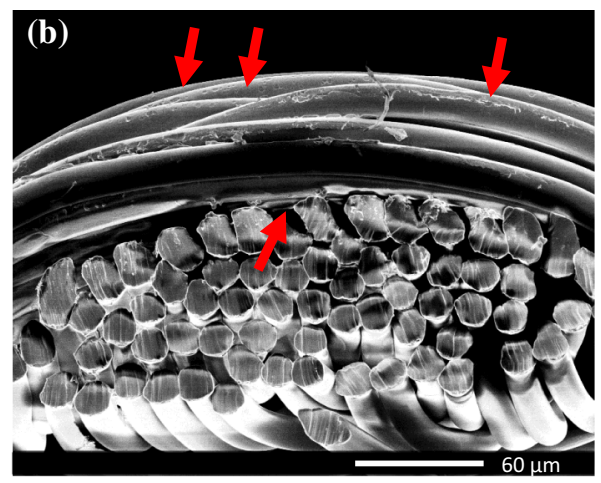

PET. b Cross-sectional view of inkjet-printed PET $\times 10$. The functional ink is deposited mostly on the top surface, as pointed by arrow.

ISO 6330 standard. During washing, the functional surface is exposed thoroughly to agitation by detergent, mechanical vibration and elevated temperature. As plotted in Fig. 4a, the PET $\times 10$ and PA $\times 10$ samples preserved similar level of WCA after 10 wash cycles. The WCA of PET $\times 10$ was $136^{\circ}$ and became $133^{\circ}$; the WCA of PA $\times 10$ was $137^{\circ}$ and became $131^{\circ}$ after 10 wash cycles. In comparison, the reference PET with fluorocarbon finishing has a decreased WCA from $143^{\circ}$ to $126^{\circ}$ whereas the reference PA with fluorocarbon finishing has a decreased WCA from $140^{\circ}$ to $128^{\circ}$ after 10 wash cycles. The results showed the functional layer demonstrated resistance to wash. The WCA almost stayed the same after 10 wash cycles.

SEM analysis was performed on PET $\times 10$ and PA $\times 10$ samples after 1 and 10 wash cycles. After one wash, there are spherical-shaped particles and agglomerated clusters of such particles formed at the functional surface for both PET $\times 10$ and PA $\times 10$ samples (Fig. 5b, e). The formed particle has a diameter smaller than $5 \mu \mathrm{m}$ after one wash but became progressively denser and tended to form clusters larger than $10 \mu \mathrm{m}$ with various shapes and dimensions after 10 wash cycles (Fig. 5c, f). A tenable assumption is that the washing process abraded the functional surface and partially rubbed off some functional material to form the spherical particles. As shown in Fig. $5 \mathrm{i}, \mathrm{O}, \mathrm{Si}, \mathrm{Na}$ and $\mathrm{Ca}$ were the main elements detected on PET $\times 10 \_$Wash $\times 10$ sample. The detection of $\mathrm{O}$ and $\mathrm{Si}$ elements suggested that the functional ink remains at the textile surface. The $\mathrm{Na}$ and $\mathrm{Ca}$ could be residuals from detergents added during wash tests (referring to surfactant, zeolites, 


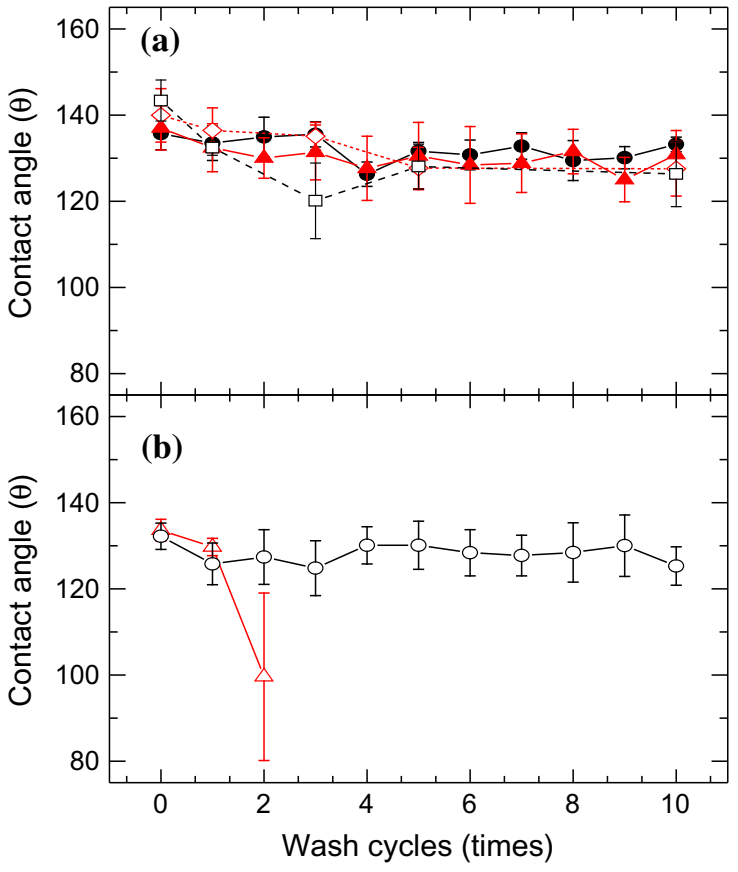

Figure 4 Water contact angle of inkjet functionalized PET and PA samples after various wash and abrasion cycles. a The inkjet functionalized PET $\times 10$ (black circles) and $\mathrm{PA} \times 10$ (red triangles) samples were washed up to 10 cycles; reference PET (open squares) and PA (open red diamonds) with fluorocarbon finishing were presented. $\mathbf{b}$ The inkjet functionalized PET $\times 1$ (open circles) and $\mathrm{PA} \times 1$ (open red triangles) samples were washed 10 and 2 cycles, respectively. $\mathrm{c}$ The inkjet functionalized PET $\times 10$ (black circles), PA $\times 10$ (red triangles), untreated PET

etc., as component in the washing detergent). The proportion of elements is not shown since EDX is a qualitative method. Furthermore, element mapping of $\mathrm{Si}$ confirmed that $\mathrm{Si}$ accumulated locally on a few aggregated spherical particles on one filament of PET $\times 10$ _Wash $\times 10$ samples (SI, Fig. S5b). This suggested that the functional ink was mechanically abraded, chemically agitated and thereafter formed spherical particles, which stick to the textile substrate by physical interaction, or are partially removed (damaged) from the filament. As shown in Fig. $5 \mathrm{~g}$ (see arrow), the washing process might attack the functional layer in a similar way like peeling an onion, i.e., layer by layer. The textile could preserve the hydrophobicity as long as there is some functional material wrapped around/sticking to the filament, regardless if it is a form of flat layer or in other spherical-like shapes. However, the change in microstructure indicated damage or disturbance of the inkjet-printed functional layer. Various liquids

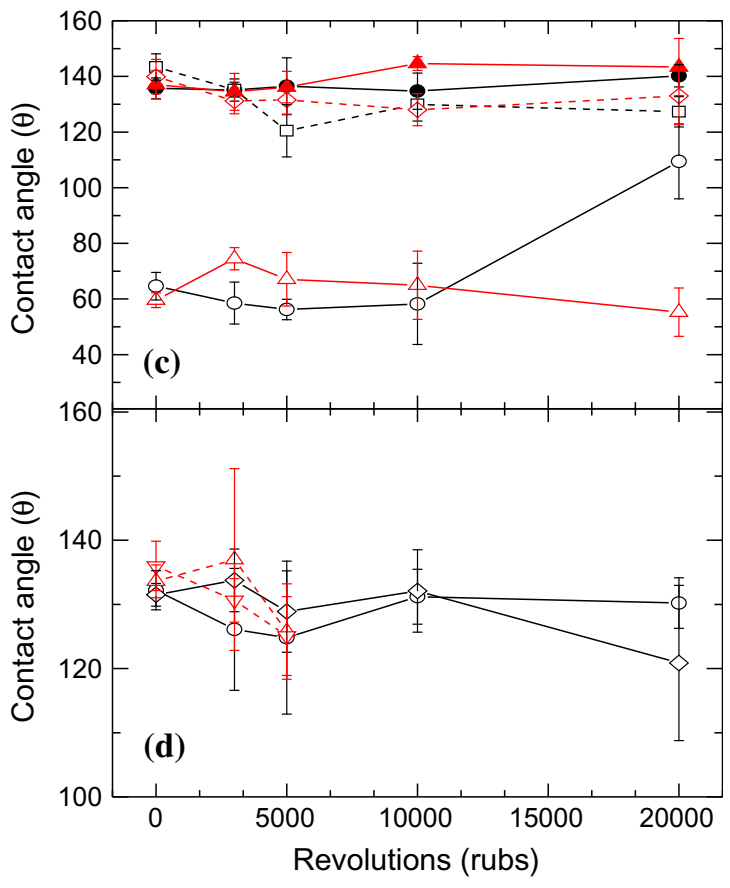

(open circles), untreated PA (open triangles) samples, reference PET (open squares) and PA (open red diamonds) with fluorocarbon finishing; $\mathbf{d}$ The inkjet functionalized PET $\times 1$ (open circles), PET $\times 3$ (open diamonds), PA $\times 1$ (open red upward triangles) and PA $\times 3$ (open red downward triangles) were rubbed up to 20000 revolutions. The error bar is the standard deviation from five independent measurements, and the connection solid and dash lines are to guide the eyes.

were placed on a PET $\times 10 \_$Wash $\times 10$ samples to verify the water repellency after washing. As shown in Fig. 2e, the inkjet-printed area of PET $\times 10_{-}$ Wash $\times 10$ sample showed repellency to water, coffee and blue-colored ink. However, we noticed that the time for the textile to hold the water droplet after 10 washes is reduced. The WCA started to decrease after drop placement within 3-5 min. The deterioration of water repellency of the functional layer agreed with the damage of the functional layer in microstructure. The durability of the functional layers on textile is challenging and demanding. Similar comparison can be made with functional layers produced with other methods, and Vasiljevic et al. [35] produced a hierarchically roughened surface with $\mathrm{SiO}_{2}$ nanoparticles covered by fluoroalkyl functional oligosiloxane by pad-dry-cure method. During wash fastness testing, the functional layer starts to change after 10 domestic washes. Schwarz et al. [36] produced thin copper films on para-aramid yarns via 

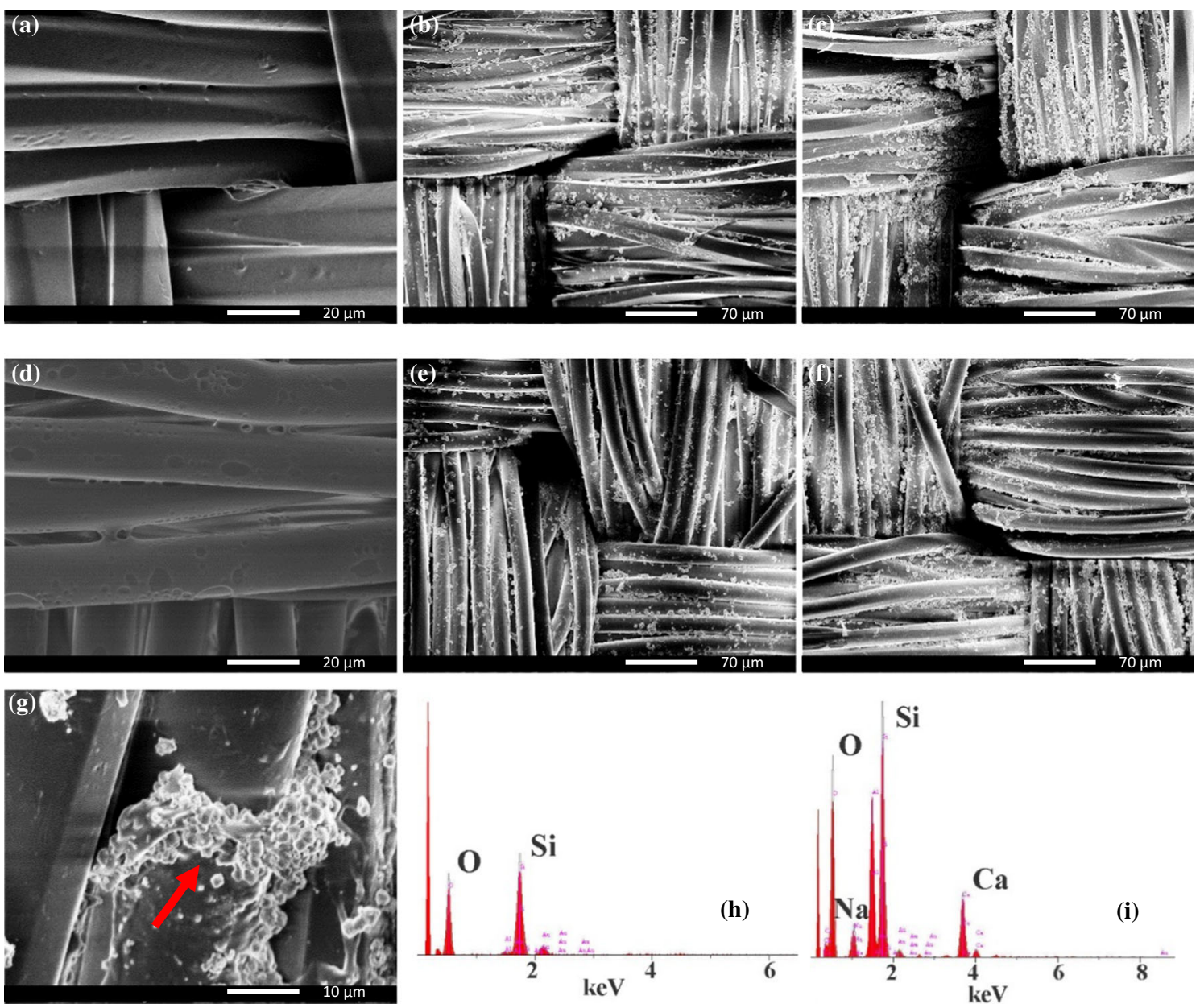

Figure 5 SEM pictures of inkjet-printed PET and PA after various wash cycles and EDX spectra. PET $\times 10$ sample with a after inkjet printing, $\mathbf{b}$ after one wash cycle, $\mathbf{c}$ after 10 wash cycles; and PA $\times 10$ sample $\mathbf{d}$ after inkjet printing, e after one wash cycle,

wet chemistry route. The morphology of yarns shows damage after 25 washes. In both examples, the functional properties such as water and oil repellency or electrical conductivity deteriorated with damaged functional layers after washing. Introduction of a cross-linking mechanism in the ink formulation could reinforce the functional layer on textile as suggested by Liu et al. [1] and Zhou et al. [28]. Still, even though the polysiloxane used in this study could release to environment, it is a linear no-violate type material which is rather safe $[26,37]$. The material is intended to be deposited on the outside surface of garment which has less direct contact with the human skin.

The functionalized PET showed better wash fastness property due to (a) the stronger ink-filament interaction with PET, and (b) the deeper transportation of the functional ink in PET. As shown in Fig. $4 \mathrm{~b}$, f after 10 wash cycles. $\mathbf{g}$ PET $\times 10 \_$Wash $\times 5$ sample showed peeling of functional materials and formation of particles/clusters (see arrow). $\mathbf{h}$ EDX analysis of PA $\times 10$ sample and $\mathbf{i}$ EDX analysis of PET $\times 10_{\text {_Wash }} \times 10$ sample.

the WCA of PET $\times 1$ was $132^{\circ}$ and became $125^{\circ}$ after 10 wash cycles. But the WCA of PA $\times 1$ became $<$ $90^{\circ}$ after 2 wash cycles from an initial WCA of $134^{\circ}$. The PET $\times 1$ shows better wash fastness than PA $\times 1$ sample. This could be a result of weak interaction between the functional ink and PA filament. As shown in liquid absorption tests of untreated PET and PA (SI, Table S1), the untreated PA absorbed slightly more high surface tension liquid, e.g., water [38] $\left(72.09 \mathrm{mN} \mathrm{m}^{-1}\right)$ but less low surface tension liquid, e.g., acetonitrile and ethyl acetate, than untreated PET. The surface tension of the functional ink $\left(23.9 \mathrm{mN} \mathrm{m}^{-1}\right)$ is very similar to acetonitrile $\left(27.76 \mathrm{mN} \mathrm{m}^{-1}\right) \quad$ [38] and ethyl acetate (24.11 $\mathrm{mN} \mathrm{m}^{-1}$ ) [39]. This means the PET might have stronger ink-filament interaction than PA due to better wettability with the functional ink. 
Furthermore, transportation of the functional ink is assessed theoretically and experimentally. The Washburn-Lucas equation [40] describes the transportation of liquid within a two-end open pore with external pressure opposing capillary flow. By assuming the opposition pressure is negligible, the equation became:

$l=\sqrt{\left(\frac{r \cdot \gamma \cdot \cos \theta}{2 \cdot \eta}\right) \cdot t}$

where $l$ is the distance penetrated by a liquid, $r$ is the effective capillary radius of the pores, $\gamma$ is the surface tension of the applied liquid, $\theta$ is the liquid/pore contact angle, $\eta$ is the viscosity of the liquid and $t$ is the time. The wicking property of the fabric was assessed by the absorption rate of the ink on the substrate (SI). As shown in Fig. S2, the absorption of the functional ink on PET is more or less linear in speed as in Eq. (1), and faster than the absorption on PA. Furthermore, the absorption of the functional ink on PA is a two-stage or exponential decay process. The ink was absorbed faster at the beginning but got slower with time. Moreover, in capillary height measurement in warp and weft direction of untreated PA and PET, the absorption rate of water in untreated PET is higher than PA (SI, Fig. S1). The results suggested that untreated PET could have larger pores, i.e., inter- and intra-yarn spaces $r$, than PA in Eq. (1); therefore, larger $l_{\mathrm{PET}}$ was observed experimentally (assuming $\cos \theta_{\mathrm{PET}} \approx \cos \theta_{\mathrm{PA}}$ ). As a result, the ink spread more on the surface of PA rather than transporting it deeper. This could be deducted by experimentally measured smaller $r_{\mathrm{PA}}$ (average pore size of PA of $11.2 \mu \mathrm{m}<\mathrm{PET}$ of $17.7 \mu \mathrm{m})$ and $l_{\mathrm{PA}}$ from Eq. (1). Finally, this assumption is supported by SEM picture of PA $\times 10$ sample, where the functional ink accumulated more on the PA top surface and therefore is more vulnerable to washing. Overall, the functional ink absorption measurement, capillary height measurement and pore size measurement suggested coherent evidences that the ink is transported deeper in PET. Liu et al. [1] studied the hydrophobicity and durability of fluorocarbon knifecoated cotton. The WCA of fluorocarbon-coated cotton is $\sim 20^{\circ}$ (WCA of $\sim 153^{\circ}$ ) higher than inkjetprinted polysiloxane on PET or PA. The WCA of noncrosslinked fluorocarbon coating decreased to $144^{\circ}$ after 10 wash cycles [1], similarly as decrease in
WCA $\left(137^{\circ}\right.$ to $\left.\sim 132^{\circ}\right)$ in our observation. Furthermore, polysiloxane padded [41] or grafted to cotton fabrics [42] can improve the WCA from $<90^{\circ}$ to $120^{\circ}-130^{\circ}$. Polysiloxane combined inkjet printing shows the capability to functionalize a water-repellent surface as a more environmentally friendly alternative.

The durability of the functional layer toward abrasion was tested by Martindale tester according to modified ISO 12947-2:1998/Cor.1:2002 standard. As shown in Fig. $4 \mathrm{c}$, the PET $\times 10$ and PA $\times 10$ samples preserved the hydrophobicity after 20000 revolutions. The WCA of PET $\times 10$ was $136^{\circ}$ before and became $140^{\circ}$ after rubbing; the WCA of PA $\times 10$ was $137^{\circ}$ and remained $143^{\circ}$ after 20000 rubs. In comparison, the untreated PET and PA samples showed no improved WCA after abrasion tests. The SEM images (Fig. 6) suggested that the functional layer was not affected very much in both PET $\times 10$ and PA $\times 10$ samples after 20000 rubs. The microstructure is significantly different compared with inkjet functionalized textiles after wash fastness tests. Furthermore, the reference PET had a slightly decreased WCA from $143^{\circ}$ to $127^{\circ}$, whereas the reference PA remained the hydrophobicity from $140^{\circ}$ to $133^{\circ}$ after 20000 rubs. We noticed that the abrasion tests could produce a fluffy surface due to abrasion of textile substrate against the abrading material. At microscopic level, the inkjet functionalized textile became planar functionalized woven yarns with protruding filaments with functional wrapping. According to Cassie-Baxter equation $[43,44]$,

$\cos \theta_{\mathrm{CB}}=f_{\mathrm{s}} \cdot\left(\cos \theta_{Y}+1\right)-1$

where $\theta_{\text {Св }}$ is the Cassie-Baxter contact angle on the rough surface, the $f_{\mathrm{s}}$ is the fractions of the solid area divided by the projected surface area on the surface, and $\theta_{Y}$ is the contact angle on smooth surface. Therefore, WCA of a surface is affected by the surface roughness. These small protruding filaments with hydrophobic functionalization on the top surface may boost the hydrophobicity by reducing $f_{\mathrm{s}}$ (higher WCA $\theta_{\mathrm{CB}}$ in Eq. 2). The resistance to abrasion of inkjet functionalized samples is comparable or slightly better than fluorocarbon-finished reference PET and PA. Abrasion tests were performed on PET $\times 1$, PET $\times 3$, PA $\times 1$ and PA $\times 3$ samples. As plotted in Fig. 4 d, PET $\times 1$ and PET $\times 3$ samples have WCA of $130^{\circ}$ and $121^{\circ}$ after 20000 rubs. But 
Fig. 6 SEM images of $\mathrm{PET} \times 10$ and PA $\times 10$ samples with 20000 rubs after abrasion tests. PET $\times 10$ sample with a $1000 \times$ and b $3000 \times$ magnification; and PA $\times 10$ sample with c $1000 \times$ and d $3000 \times$ magnification.
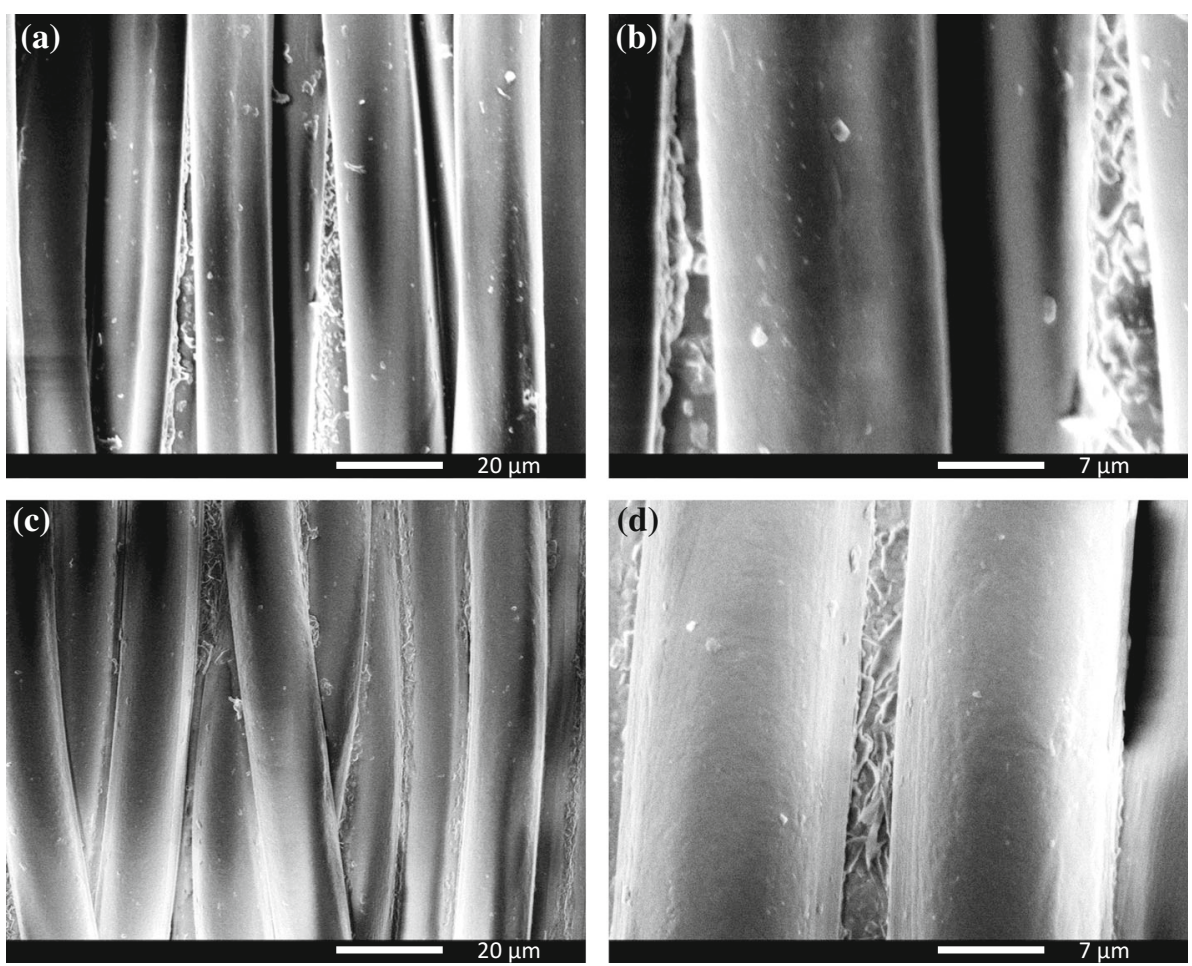

PA $\times 1$ and PA $\times 3$ samples became hydrophilic after 10000 and 20000 rubs. The PET $\times 1$ and PET $\times 3$ samples showed better abrasion resistance compared to $\mathrm{PA} \times 1$ and $\mathrm{PA} \times 3$ samples. The results suggested that the functional ink adhered better/interacted stronger with PET. However, the capability of samples to hold water droplets decreased significantly, suggesting damage of the functional layer. After 3000 rubs, the droplets can wet $\mathrm{PET} \times 1, \mathrm{PET} \times 3, \mathrm{PA} \times 1$ and $\mathrm{PA} \times 3$ samples within $5 \mathrm{~min}$, despite a high initial WCA.

The ink transportation and ink-filament interaction are crucial to understand the durability of the functional layer in abrasion. Two factors, porosity/effective pore size of the textile substrate and ink-filament interaction played a key role. As shown experimentally in capillary height and functional ink absorption measures in Fig. S1 and S2, as well as theoretical deduction in Eq. (1), the functional ink penetrates deeper in PET fabrics. This can be the reason to obtain better abrasion resistance with PET. The inkjetprinted ink first covered the top layer of the textile surface and thereafter transported deeper through inter- and intra-yarn spaces into the bulky textiles. The ink transported deeper into the textile could offer better resistance, whereas functional ink accumulated on the top of the surface is more vulnerable to abrasion. Furthermore, PET indicates stronger ink-filament interaction with the functional ink. The functional ink adhered better on PET $\times 1$ and $\times 3$ samples than PA $\times 1$ and $\times 3$ samples, which agreed with previous wash fastness results. Moreover, the filaments wrapped around with a thicker layer of functional material are more resistant to abrasion. As shown in Fig. $4 d$, the sample with less functional material $\left(\mathrm{PET} \times 1, \times 3\right.$ and PA $\times 1, \times 3,<1 \mathrm{~g} \mathrm{~m}^{-2}$ ) is more vulnerable to abrasion tests than PET $\times 10$ and PA $\times 10$ samples $\left(1.6 \mathrm{~g} \mathrm{~m}^{-2}\right)$. Overall, the inkjetprinted PET and PA surface with polysiloxane showed moderately high WCA, wash fastness and abrasive property. However, due to the nature of non-covalent interaction between the functional layer and textile substrate, the fastness properties are constrained. To improve water repellency, as well as fastness properties, it could be interesting to introduce covalent bonding between the functional layer and textile substrates in combination with nanoscale surface roughening. 


\section{Conclusion}

Waterborne, fluorocarbon-free and water-repellent ink with polysiloxane micro-emulsion was formulated and inkjet printed on plain woven PET and PA textile substrates, in order to functionalize the textile with water repellency. The PET and PA have a WCA from $<90^{\circ}$ to $140^{\circ}$ after inkjet functionalization. The functional textile has a microstructure with polysiloxane wrapped around the filament which modified the surface property of the textiles. Furthermore, the functional surfaces show resistance to wash and abrasion. The 10-pass printed PET $($ PET $\times 10)$ preserved a WCA of $133^{\circ}$ after 10 washes and a WCA of $140^{\circ}$ after 20000 rubs. We found that the ink transportation in the textile structure as well as the ink-filament interaction plays a key role in the durability of the functional layer. The functional ink was transported faster and deeper in PET, and with stronger non-covalent ink-filament interaction with PET than with PA. Therefore, PET showed better wash fastness and abrasion resistance. The microscopic images showed that inkjet printing did not or had little impact on the tactile feeling of the textile, i.e., the hand feeling and softness of textile is well preserved. Inkjet printing demonstrated selective and efficient deposition of functional material on textiles, as a promising resource-efficient process in the applications of functional and smart textiles.

\section{Acknowledgements}

The authors are grateful for the support from CROSSTEXNET, Digifun project, KK Stiftelsen (The Knowledge Foundation) with Diarienummer: 20150040, TEKO (The Swedish Textile and Clothing Industries Association), SST Stiftelsen (The foundation for Swedish Textile Research), Myfab, Borås stad and Sparbanksstiftelsen Sjuhärad for enabling this research.

\section{Compliance with ethical standards}

Conflict of interest The authors declare that they have no conflict of interest.

Electronic supplementary material: The online version of this article (https://doi.org/10.1007/ s10853-018-2521-z) contains supplementary material, which is available to authorized users.
Open Access This article is distributed under the terms of the Creative Commons Attribution 4.0 International License (http://creativecommons.org/ licenses/by/4.0/), which permits unrestricted use, distribution, and reproduction in any medium, provided you give appropriate credit to the original author(s) and the source, provide a link to the Creative Commons license, and indicate if changes were made.

\section{References}

[1] Liu Y, Xin JH, Choi C-H (2012) Cotton fabrics with singlefaced superhydrophobicity. Langmuir 28:17426-17434

[2] Hoth CN, Choulis SA, Schilinsky P, Brabec CJ (2007) High photovoltaic performance of inkjet printed polymer: fullerene blends. Adv Mater 19:3973-3978

[3] Korvink JG, Smith PJ, Shin D-Y (2012) Inkjet-based Micromanufacturing. Wiley-VCH, Weinheim

[4] Tekin E, Smith PJ, Schubert US (2008) Inkjet printing as a deposition and patterning tool for polymers and inorganic particles. Soft Matter 4:703-713

[5] spgprints (2017) http://www.spgprints.com/textile+printing/ systems+textile/systems/digital+printing+systems/pike? product_id=614. Accessed 13 June 2018

[6] Layani M, Cooperstein I, Magdassi S (2013) UV crosslinkable emulsions with silver nanoparticles for inkjet printing of conductive $3 \mathrm{D}$ structures. J Mater Chem C 1:3244-3249

[7] Tang W, Feng L, Zhao J, Cui Q, Chen S, Guo X (2014) Inkjet printed fine silver electrodes for all-solution-processed low-voltage organic thin film transistors. J Mater Chem C 2:1995-2000

[8] Belgardt C, Sowade E, Blaudeck T, Baumgartel T, Graaf H, von Borczyskowski C, Baumann RR (2013) Inkjet printing as a tool for the patterned deposition of octadecylsiloxane monolayers on silicon oxide surfaces. Phys Chem Chem Phys 15:7494-7504

[9] Del Mauro ADG, Diana R, Grimaldi IA, Loffredo F, Morvillo P, Villani F, Minarini C (2013) Polymer solar cells with inkjet-printed doped-PEDOT: PSS anode. Polym Compos 34:1493-1499

[10] Zhuang J-L, Ar D, Yu X-J, Liu J-X, Terfort A (2013) Patterned deposition of metal-organic frameworks onto plastic, paper, and textile substrates by inkjet printing of a precursor solution. Adv Mater 25:4631-4635 
[11] Roach P, Shirtcliffe NJ, Newton MI (2008) Progess in superhydrophobic surface development. Soft Matter 4:224-240

[12] Vilčnik A, Jerman I, Šurca Vuk A, Koželj M, Orel B, Tomšič B, Simončič B, Kovač J (2009) Structural properties and antibacterial effects of hydrophobic and oleophobic sol-gel coatings for cotton fabrics. Langmuir 25:5869-5880

[13] Nechyporchuk O, Yu J, Nierstrasz VA, Bordes R (2017) Cellulose nanofibril-based coatings of woven cotton fabrics for improved inkjet printing with a potential in E-textile manufacturing. ACS Sustain Chem Eng 5:4793-4801

[14] Xue C-H, Jia S-T, Zhang J, Ma J-Z (2010) Large-area fabrication of superhydrophobic surfaces for practical applications: an overview. Sci Technol Adv Mater 11:033002

[15] Gulrajani ML (2013) Advances in the dyeing and finishing of technical textiles. Woodhead Publishing, Cambridge

[16] Whittow WG, Chauraya A, Vardaxoglou JC, Li Y, Torah R, Yang K, Beeby S, Tudor J (2014) Inkjet-Printed microstrip patch antennas realized on textile for wearable applications. IEEE Antenna Wirel Propag Lett 13:71-74

[17] Takamatsu S, Lonjaret T, Crisp D, Badier J-M, Malliaras GG, Ismailova E (2015) Direct patterning of organic conductors on knitted textiles for long-term electrocardiography. Sci Rep 5:15003

[18] Hoath SD (2015) Fundamentals of inkjet printing. Wiley, Berlin

[19] Yao X, Song Y, Jiang L (2011) Applications of bio-inspired special wettable surfaces. Adv Mater 23:719-734

[20] Zeng C, Wang H, Zhou H, Lin T (2015) Self-cleaning, superhydrophobic cotton fabrics with excellent washing durability, solvent resistance and chemical stability prepared from an SU-8 derived surface coating. RSC Adv 5:61044-61050

[21] Karim N, Afroj S, Malandraki A, Butterworth S, Beach C, Rigout M, Novoselov KS, Casson AJ et al (2017) All inkjetprinted graphene-based conductive patterns for wearable E-textile applications. J Mater Chem C 5:11640-11648

[22] Yanhua L, Zhilu L, Yupeng L, Haiyuan H, Yi L, Pengxun Y, Bo Y, Feng Z (2015) One-step modification of fabrics with bioinspired polydopamine@octadecylamine nanocapsules for robust and healable self-cleaning performance. Small 11:426-431

[23] Wang Z, Cousins IT, Scheringer M, Hungerbühler K (2013) Fluorinated alternatives to long-chain perfluoroalkyl carboxylic acids (PFCAs), perfluoroalkane sulfonic acids (PFSAs) and their potential precursors. Environ Int 60:242-248

[24] (2017) Commission regulation (EU) 2017/1000, amending Annex XVII to Regulation (EC) No 1907/2006 of the European Parliament and of the Council concerning the
Registration, Evaluation, Authorisation and Restriction of Chemicals (REACH) as regards perfluorooctanoic acid (PFOA), its salts and PFOA-related substances

[25] McCann J (2015) Textiles for sportswear. Woodhead Publishing, Cambridge

[26] Mojsiewicz-Pieńkowska K, Jamrógiewicz M, Szymkowska K, Krenczkowska D (2016) Direct human contact with siloxanes (silicones) - safety or risk part 1. Characteristics of siloxanes (silicones). Front Pharmacol 7:132

[27] Teisala H, Tuominen M, Kuusipalo J (2014) Superhydrophobic coatings on cellulose-based materials: fabrication, properties, and applications. Adv Mater Interfaces 1:1300026

[28] Zhou H, Wang H, Niu H, Gestos A, Wang X, Lin T (2012) Fluoroalkyl silane modified silicone rubber/nanoparticle composite: a super durable, robust superhydrophobic fabric coating. Adv Mater 24:2409-2412

[29] Wheeler JSR, Reynolds SW, Lancaster S, Romanguera VS, Yeates SG (2014) Polymer degradation during continuous ink-jet printing. Polym Degrad Stab 105:116-121

[30] ASTM D5725-99 (2008) Standard test method for surface wettability and absorbency of sheeted materials using an automated contact angle tester

[31] Sharma S, Pande SS, Swaminathan P (2017) Top-down synthesis of zinc oxide based inks for inkjet printing. RSC Adv 7:39411-39419

[32] Jin M, Feng X, Xi J, Zhai J, Cho K, Feng L, Jiang L (2005) Super-hydrophobic PDMS surface with ultra-low adhesive force. Macromol Rapid Commun 26:1805-1809

[33] Malm V, Walkenström P (2015) Influence of rheology modifiers and coating parameters on the color-changing effects of textile coatings with multi-layered mica pigments. Text Res J 85:936-948

[34] Åkerfeldt M, Strååt M, Walkenström P (2013) Electrically conductive textile coating with a PEDOT-PSS dispersion and a polyurethane binder. Text Res J 83:618-627

[35] Vasiljević J, Zorko M, Tomšič B, Jerman I, Simončič B (2016) Fabrication of the hierarchically roughened bumpysurface topography for the long-lasting highly oleophobic "lotus effect" on cotton fibres. Cellulose 23:3301-3318

[36] Schwarz A, Hakuzimana J, Westbroek P, Mey GD, Priniotakis G, Nyokong T, Langenhove LV (2012) A study on the morphology of thin copper films on para-aramid yarns and their influence on the yarn's electro-conductive and mechanical properties. Text Res J 82:1587-1596

[37] Pieńkowska KM (2014) Concise encyclopedia of high performance silicones. Wiley, London

[38] Rafati AA, Bagheri A, Najafi M (2010) Experimental data and correlation of surface tensions of the binary and ternary systems of water + acetonitrile + 2-propanol at $298.15 \mathrm{~K}$ and atmospheric pressure. J Chem Eng Data 55:4039-4043 
[39] JdlS López-Lázaro, Iglesias-Silva GA, Estrada-Baltazar A, Barajas-Fernández J (2015) Density and surface tension of binary mixtures of 2,2,4-trimethylpentane $+n$-heptane, 2,2,4-trimethylpentane + n-octane, ethyl acetate + benzene, and butanenitrile + benzene from (293.15 to 323.15) K. J Chem Eng Data 60:1823-1834

[40] Washburn EW (1921) The dynamics of capillary flow. Phys Rev 17:273-283

[41] Dong C, Lu Z, Zhang F, Zhu P, Zhang L, Sui S (2015) Preparation and properties of cotton fabrics treated with a novel polysiloxane water repellent and flame retardant. Mater Lett 152:276-279

[42] Izmailov BA, Nedel'kin AV, Rodlovskaya EN, Zachernyuk BA, Nedel'kin VI (2012) Molecular assembly of nanolayers of water-repellent siloxane coatings on the surface of fibers. Fibre Chem 43:433-435

[43] Sigmund WM, Hsu S-H (2016) In: Drioli E, Giorno L (eds) Encyclopedia of membranes. Springer, Berlin

[44] Bormashenko E (2008) Why does the Cassie-Baxter equation apply? Colloids Surf A 324:47-50 